\title{
Increased Operational Availability and Simplified Operations Using Dither Gyro Scale Factor Calibration
}

\author{
Alan D. Reth ${ }^{1}$ \\ Chesapeake Aerospace, LLC, Grasonville, MD, USA, alan.d.reth@nasa.gov \\ Dr. Delano R. Carter ${ }^{2}$ \\ Thearality, Inc, Glendale, AZ, USA, delano.r.carter@nasa.gov \\ Douglas C. Freesland ${ }^{3}$ \\ The ACS Engineering Corporation, Clarksville, MD, USA, douglas.c.freesland@nasa.gov \\ and \\ Alexander Krimchansky ${ }^{4}$ \\ NASA Goddard Space Flight Center, Greenbelt, MD, USA, alexander.krimchansky@nasa.gov
}

\begin{abstract}
The traditional approach to on-orbit gyro scale factor calibration has been to perform large angle rotations about each gyro axis. The maneuvers require the science instruments to be taken offline, reducing operational availability and require a significant amount of interaction from the ground. To increase operational availability and to reduce the burden on mission operators, a novel approach to gyro scale factor calibration was developed, modeled and successfully demonstrated on the Geostationary Operational Environmental Satellite (GOES-16) to estimate gyro scale factor errors to within 1500 parts per million (ppm) without taking the science instruments offline.
\end{abstract}

\section{Introduction}

High-precision space missions may require accurate gyro scale factor knowledge in order to meet requirements. Gyro scale factor is measured to within required accuracy prior to spacecraft launch and typically again during onorbit commissioning. However, gyro scale factors drift over time, which can result in non-compliant pointing control and knowledge. Periodic calibration of gyro scale factor over the life of a mission may be necessary in order to meet requirements.

One traditional method to calibrate gyro scale factor is to add scale factor states to a standard six-state attitude determination Kalman Filter. For mission profiles that include spacecraft slews that are large enough and frequent enough, this may be a viable approach. For missions with attitude profiles that are mostly steady-state, for example, inertially fixed or rotating at one revolution per orbit as in a nadir-pointing mission, gyro scale factor errors may not be adequately observable for accurate estimation via Kalman Filter. Another traditional method overcomes the observability problem by executing large-angle spacecraft slews for gyro calibration, making it possible to distinguish the effects of gyro scale factor from gyro bias. Under that approach, data acquired during large calibration slews may be processed by an onboard Kalman Filter or on the ground to estimate the gyro calibration parameters. This method requires taking the spacecraft offline, temporarily suspending normal operations for calibration maneuvers. When scale factor recalibration is required periodically throughout a mission, one can be faced with a choice between loss of availability or degradation of pointing performance due to gyro scale factor drift.

In this paper, we present a new methodology for estimating gyro scale factor during normal spacecraft operations, using small attitude motions that are compliant with mission pointing requirements and a signal processing method

\footnotetext{
${ }^{1}$ NASA Goddard Space Flight Center, GOES-R Flight, Image Navigation and Registration.

${ }^{2}$ NASA Goddard Space Flight Center, GOES-R Flight, Image Navigation and Registration, Senior Member AIAA.

${ }^{3}$ NASA Goddard Space Flight Center, GOES-R Flight, Guidance Navigation and Control.

${ }^{4}$ NASA Goddard Space Flight Center, GOES-R Flight, System Manager and Chief Architect.
} 
that specifically detects the intentionally induced motions, first described by Reth et al. 2011 [1]. Small, intentionally induced attitude motions are hereinafter referred to as dither. The calibration technique uses sinusoidal dither profiles of a frequency and amplitude that are designed such that attitude error, attitude rate, attitude stability, and reaction wheel torque allocations are simultaneously satisfied, enabling science instruments to continue normal operations throughout the calibration period.

A Monte Carlo analysis was performed in 2011 using a low-fidelity simulation to quantify dither scale factor calibration accuracy in the presence of realistic gyro and star tracker noise. The results demonstrated the feasibility of estimating gyro scale factor to an accuracy better than 2500 parts per million (ppm). In addition, a blind test was performed in which the Geostationary Operational Environmental Satellite R-Series (GOES-R) spacecraft vendor provided two data sets from a high-fidelity attitude control system (ACS) simulation that included gyro and star tracker noise, instrument-induced disturbances, and spacecraft flexible-body modes. The two data sets each had a different scale factor error intentionally introduced into the gyro model in simulation runs of the spacecraft executing dither excitation. The GOES-R Project team used their dither calibration processing algorithm to recover the unknown scale factor errors to an accuracy better than $1400 \mathrm{ppm}$ in one case and 400 in the other.

In 2016 and 2017, the dither calibration technique was successfully demonstrated to estimate gyro scale factor errors on the GOES $-16^{*}$ spacecraft. The on-orbit dither results were compared to a pair of more traditional methods using 19 degree and 3.5 degree spacecraft slews about each gyro axis. The results demonstrate the ability of the dither technique to estimate gyro scale factor errors on-orbit to better than $1500 \mathrm{ppm}$.

\section{GOES-R Description}

GOES-R is the first in a series of next generation U.S. geostationary weather satellites built by Lockheed Martin Space System and managed jointly by the National Oceanic and Atmospheric Administration (NOAA) and the National Aeronautics and Space Administration (NASA). The GOES-R instrument suite includes two Earth pointed instruments: Advanced Baseline Imager (ABI) and Geostationary Lightning Mapper (GLM); two solar pointed instruments: Solar Ultraviolet Imager (SUVI) and Extreme Ultraviolet and X-ray Irradiance Sensors (EXIS); and two space weather instruments: Space Environment In-Situ Suite (SEISS) and a pair of magnetometers. The satellite configuration is shown in Fig. 1.

Attitude determination utilizes the Northrop Grumman Scalable Space Inertial Reference Unit (SSIRU) for the Inertial Measurement Unit (IMU), and the Sodern Hydra with three optical heads for the star tracker $[2,3]$. The design includes two SSIRUs with four gyros each, but only one SSIRU is powered on at a time. The SSIRU was chosen for its high bandwidth and low latency rate output, and for its low-noise characteristics. The four gyros are sampled at $200 \mathrm{~Hz}$, and the star tracker optical heads are sampled at $20 \mathrm{~Hz}$. Attitude estimation is performed using a kinematic

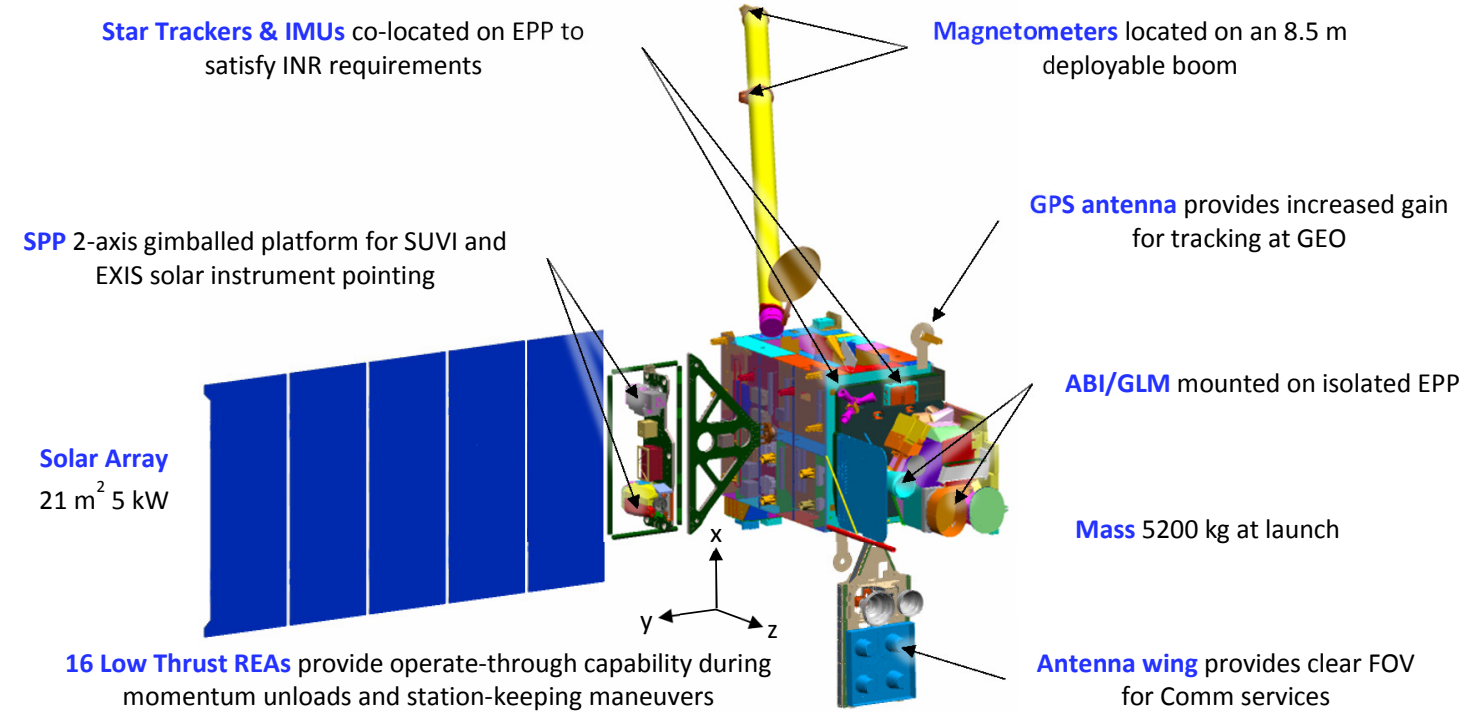

Fig. 1 The GOES-R satellite configuration.

\footnotetext{
*After achieving geostationary orbit, GOES-R was renamed GOES-16. In this paper, "GOES-R" will generally be used when discussing the design of the spacecraft series, and "GOES-16" will be used when discussing flight data.
} 
six-state extended Kalman filter, which combines quaternion outputs from the star tracker with angular rate measurements from the SSIRU to produce a three-state attitude error estimate and three-state gyro bias error estimate. Two samples of $200 \mathrm{~Hz}$ gyro data are collected, scaled, filtered, bias-corrected and converted to three-axis rate data before sending to $\mathrm{ABI}$ at $100 \mathrm{~Hz}$.

More than a decade before its launch in November 2016, system architects were focused on requirements that increased operational availability while simplifying mission operations. For example, the prior GOES series required that the instruments to be taken offline for approximately 10 minutes each day to dump momentum; undesirable especially during a critical weather event. Freesland et al. 2005 [4], showed how existing technologies could be adapted to operate-through momentum management and stationkeeping maneuvers, reducing outages by $98 \%$ from 7160 minutes per year to a new requirement of just 120 minutes per year. To accomplish this necessitated the development of a low thrust $(0.09 \mathrm{~N})$ monopropellant hydrazine thruster, dual isolation [5], and active vibration damping [6]. In addition, GOES-R includes the first use of a civilian frequency GPS receiver at geostationary orbit, providing a dramatic step forward in autonomous orbit determination performance that greatly simplifies mission operations by eliminating the need for ranging, daily ephemeris uploads, and maneuver recovery [7].

The operate-through requirement also precludes periodic large angle gyro calibration maneuvers traditionally used to separate gyro scale factor errors from bias and alignment errors. For most nadir-pointed missions this would not be an issue with scale factor error absorbed into the on-board gyro bias estimate. For GOES-R, gyro accuracy is critical for onboard instrument line-of-sight motion compensation and ground processing for Image Navigation and Registration (INR), which requires precise instrument angular rate knowledge. GOES-R INR requirements include ABI and GLM absolute image navigation of 28 and 140 microradians, respectively, and a number of ABI image registration requirements ranging down to 7 microradians, 3-sigma. ABI also uses spacecraft angular rate data for real-time scanner control to decouple instrument line of sight from spacecraft attitude motion. Instrument demands on gyro accuracy include integrated rate error requirements of 1,2, and 7 microradians, 3-sigma, over 1, 30, and 300 seconds, respectively. Integrated rate error is defined to be the integral over a specified time window of the difference between estimated and true angular rates of the spacecraft. Gyro scale factor error contributes to integrated rate error, particularly during relatively large attitude transients such as during thruster operations, when attitude motion may exceed 200 microradians. When coupled with the transients induced by thruster and instrument operations, uncompensated gyro scale factor errors could result in violations of pointing and INR performance.

\section{Dither Gyro Scale Factor Calibration}

Dither calibration of gyro scale factor starts with imparting small sinusoidal attitude motion to the spacecraft to serve as excitation that is sensed by the star trackers and gyros. Since the dither frequency is known, Fourier analysis enables robust detection of the small excitation signal in both sensors in the presence of noise. The star tracker is assumed to have zero scale factor error, so that the gyro scale factor error can be estimated as the ratio of the gyro to star tracker Fourier coefficients at the dither frequency. A simplified overview of the processing is shown in Fig. 2, where the Fourier analysis is depicted as a Fast Fourier Transform (FFT) as an example implementation, although an FFT was not used on GOES-R.

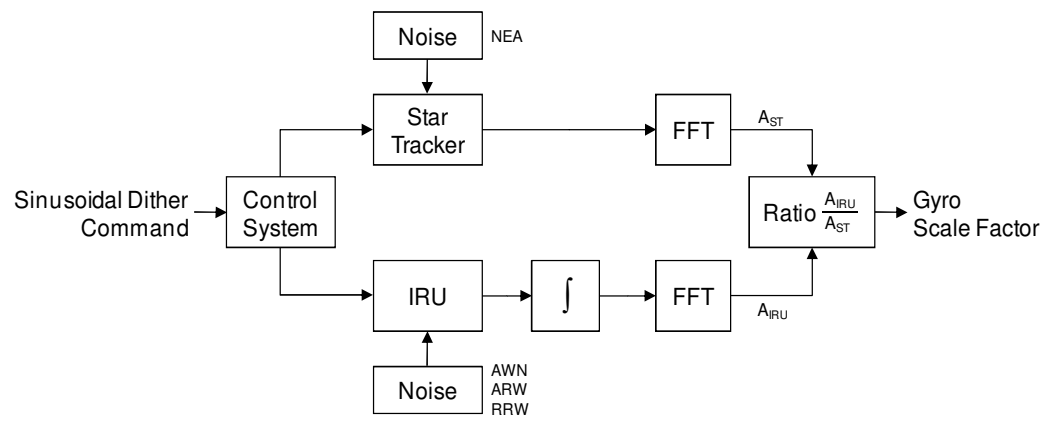

Fig. 2 Gyro scale factor estimation overview.

\section{A. Excitation Considerations}

For GOES-R attitude control requirements are somewhat generous, while attitude knowledge requirements are more stringent. Control requirements are driven primarily by science instrument coverage requirements; that is, ensuring that the instruments collect imagery from required target areas. Coverage performance is dominated by instrument alignment errors, which may be on the order of tenths of a degree (thousands of microradians). In 
comparison, the attitude control requirement of 360 microradians per axis, 3 -sigma, is a small contributor to instrument coverage while being a relatively large requirement for a precision pointing spacecraft. In contrast, the driving attitude knowledge requirements are the integrated rate error requirements levied against the gyro-based angular rate estimates provided by the spacecraft to the instruments. As mentioned, these range from 1 to 7 microradians for periods of 1 to 300 seconds.

Dither calibration intentionally degrades spacecraft attitude control for the purpose of refining attitude knowledge by way of maintaining gyro scale factor accuracy. The degraded attitude control due to dither must comply with not only the absolute attitude control requirement of 360 microradians but also the attitude rate error requirement of 78 microradians/sec and the peak-to-peak (p-p) attitude stability over 60 seconds requirement of 500 microradians. The GOES-R ACS also controls the solar array mounted Sun Pointed Platform (SPP), which supports the two solar instruments, SUVI and EXIS. SPP pointing requirements include pointing accuracy of 90 arc-seconds (436 microradians) and 60-second pointing stability of 6 arc-seconds (29 microradians). Reaction wheel torques required for dither excitation must not impair the ability of the ACS to perform its primary objectives. GOES-R has six reaction wheels, each having a torque capability of $0.35 \mathrm{Nms}$. Table I summarizes the GOES-R attitude control requirements relevant to dither excitation specification.

Table I ACS Requirements

\begin{tabular}{ll}
\hline Description & Requirement \\
\hline Attitude Control, 3-sigma & $< \pm 360 \mu \mathrm{rad}$ \\
Attitude Rate, 3-sigma & $< \pm 78 \mu \mathrm{rad} / \mathrm{sec}$ \\
Attitude Stability, p-p, 60 sec & $<500 \mu \mathrm{rad}$ \\
SPP Pointing, 3-sigma & $< \pm 90 \mathrm{arc}-\mathrm{sec}$ \\
SPP Stability, 3-sigma & $<6 \mathrm{arc}-\mathrm{sec}$ \\
Torque, per wheel & $<0.35 \mathrm{Nm}$ \\
\hline
\end{tabular}

\section{B. Dither Data Processing}

At a high level, the dither gyro scale factor calibration process imparts dither excitation to the spacecraft, computes first-order Fourier coefficients corresponding to the dither frequency for the star tracker (ST) and gyro signals, and computes the gyro scale factor as the ratio of the Fourier coefficients of the gyro to ST, assuming the ST scale factor is unity.

The nominal unit vectors of the four gyros in either of the two SSIRUs on GOES-R have spacecraft body frame components as shown in Table II. ${ }^{*}$ The spacecraft $\mathrm{x}, \mathrm{y}$, and $\mathrm{z}$ axes are controlled by the ACS to a target attitude such that $+\mathrm{z}$ axis is aligned with the nadir vector, $+\mathrm{y}$ axis is aligned with negative orbit normal, and $+\mathrm{x}$ completes the orthonormal triad, nearly aligned with spacecraft linear velocity vector. Rotations about $\mathrm{x}, \mathrm{y}$, and $\mathrm{z}$ are referred to as roll, pitch, and yaw, respectively.

Table II SSIRU Gyro Axes in Spacecraft Frame*

\begin{tabular}{ccccc}
\hline Spacecraft & \multicolumn{4}{c}{ Gyro } \\
\cline { 2 - 5 } Axis & A & B & C & D \\
\hline $\mathrm{x}$ & -1 & 1 & 1 & -1 \\
$\mathrm{y}$ & 1 & 1 & -1 & -1 \\
$\mathrm{z}$ & 1 & 1 & 1 & 1 \\
\hline
\end{tabular}

Processing gyro data for dither calibration is straightforward. The spacecraft downlinks derived angular rate from each of the four gyros, with no bias or alignment correction applied, since these corrections take place in the body frame, not on individual gyros. The four gyro rates do have an onboard scale factor estimate applied. Angular rate is derived by the ACS software since the native output of the SSIRU is whole angle. Dither processing includes resampling to a uniform time vector to remove any non-uniformity in the telemetry data, zeroth order detrending of the rates, integrating in the time domain, first order detrending of the integrated gyro rates, computing the fundamental Fourier coefficients of the result, and computing the magnitude of the first-order sine and cosine coefficients.

The spacecraft also downlinks a fused quaternion, which is a native output from the Sodern Hydra star tracker assembly (STA). This quaternion is computed by the STA processor using star measurements from all active star tracker optical heads. Dither processing of the STA quaternion starts by resampling to a uniform time vector, then

\footnotetext{
* Note that a scalar value of $1 / \sqrt{3}$ has been factored out of the unit vectors for clarity.
} 
computing a derived rate signal using a quaternion math version of a first-order back-difference. The STA derived rate is then projected onto each SSIRU gyro axis, taking into account on-orbit calibrated STA to SSIRU alignment knowledge. The STA derived rate signals about each of the gyro axes are then subjected to an identical processing chain as the gyro rate signals, resulting in a Fourier magnitude at the dither fundamental frequency.

The ratio of gyro Fourier magnitude to star tracker Fourier magnitude yields an estimate of the gyro scale factor. Note that the estimated scale factor has an ideal value of one, so the gyro to star tracker ratio minus one yields gyro scale factor error, the fractional error of the onboard gyro scale factor estimate. The onboard scale factor has units of angle per count, with an ideal value of 0.0061471 arc-sec/count, approximately equal to 0.0298 microradians/count. The additive correction implied by the estimated scale factor error is the scale factor error times the current onboard scale factor. Use of the same term, "gyro scale factor," for two distinct quantities could possibly lead to confusion, but we trust that context will make the usage clear in this paper, where in almost every case, the term refers to gyro signal scaling relative to ideal, rather than absolute weight per gyro count.

\section{GOES-16 Implementation and Flight Results}

Dither calibration was tested a number of times on the GOES-16 spacecraft during its six-month Post Launch Test (PLT) and subsequent six-month Extended Validation phases. Flight results confirmed the positive predictions of the developmental simulations, and dither calibration has been baselined for GOES-16 gyro scale factor trending.

\section{A. Square Wave Excitation}

Although early Monte Carlo simulation results showed that dither calibration was promising, there were no requirements flowed down to the spacecraft to implement dither generation. In order to test the method during GOES16 PLT, a collaborative effort amongst the Flight Project, the Mission Operations Support Team (MOST), and the spacecraft vendor, Lockheed Martin, yielded an approach to generate a periodic square wave instead of a sine wave, using existing system capabilities. This approach uses command sequences known as relative time sequences (RTS) uploaded to the spacecraft to periodically redefine reference vectors in the body frame that the attitude control system aligns with the nadir and negative orbit normal vectors during normal Earth-pointing control. The reference vectors are nominally the $+z$ and $+y$ body vectors, respectively. The RTS commands alter the reference vectors to be equal to the sum of the nominal vectors and small deviations that represent the desired square wave dither motion. In this way, periodic dither motion about any desired vector in the spacecraft frame can be commanded to be superimposed onto the normal spacecraft nadir-pointing attitude.

Based on a study performed by Lockheed Martin including high-fidelity simulation runs, the initial dither tests used of four RTSs, each inducing square wave dither motion about one of the four gyro axes of the primary SSIRU. The square wave had an amplitude of 150 microradians and a period of 500 seconds. The success criterion for this test was set at $10000 \mathrm{ppm}(1 \%)$ scale factor calibration error, with an unwritten goal of $5000 \mathrm{ppm}$. The scale factor results of the gyro calibrations using 19 degree spacecraft slews was used as the truth reference against which dither calibration accuracy was measured.

Initial results from the first square wave dither calibration test performed on December 11, 2016 met the success criterion, with a worst-case difference of $8391 \mathrm{ppm}$ between scale factor errors determined by dither versus large slews. Figure 3 shows a portion of the excitation signal from this test measured by Gyro D, including significant ACS overshoot predicted by simulations. With a sliding window average of thirteen dither periods applied to the dither results, the worst-case difference was reduced to $6460 \mathrm{ppm}$. Corrections to gyro scale factor and alignment relative to the star trackers determined by the 19 degree slews were uploaded to the spacecraft, and the large slew and dither calibrations were repeated on December 16.

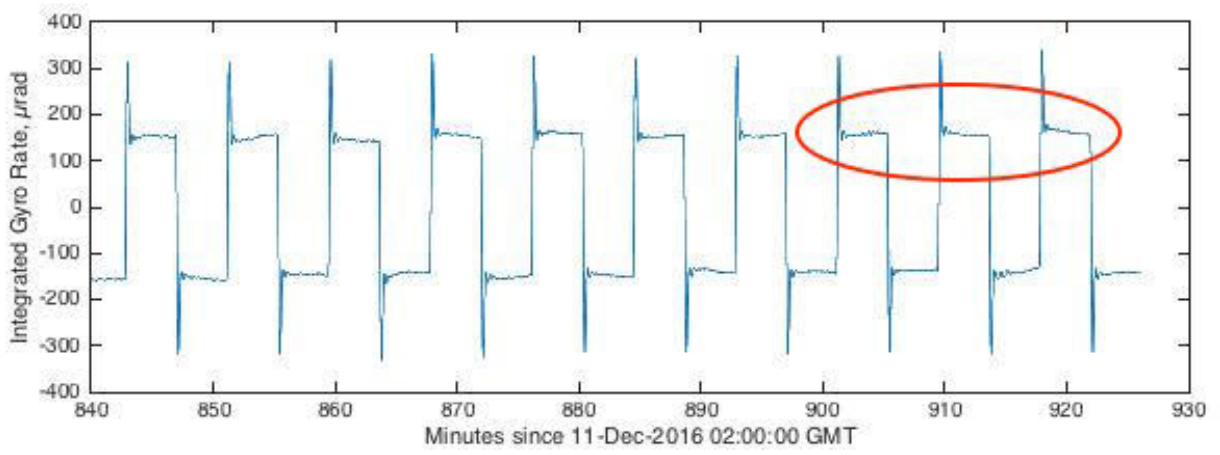

Fig. 3 Measured GOES-16 square wave dither excitation. 
For the second calibrations, the large slew measured a worst-case gyro scale factor error of $53 \mathrm{ppm}$, providing an indication of the uncertainty of that method. Worst-case scale factor error estimated by the dither method using the 13-period sliding window average was $4083 \mathrm{ppm}$. Both runs of the dither calibration passed the generous PLT success criterion of $10000 \mathrm{ppm}$, and the second test met the unwritten goal of $5000 \mathrm{ppm}$, but the results were not in line with expectations from pre-launch simulations. The method was believed to be capable of doing better.

For the dither results discussed thus far, only the on-axis gyro scale factor error was estimated for each of the four excitations, each of which was aligned with one of the four gyros in the primary SSIRU. Since the gyro axes are not orthogonal, excitation about one of the gyro axes provides incidental excitation about three gyro axes at a level of onethird of the on-axis amplitude. Although dither calibration data from the off-axis gyros has only $11 \%$ of the signal-tonoise ratio of the on axis gyro, gyro scale factor estimates for all four gyros were computed for each of the four excitations for the December 11 and 16 dither tests. The rationale was that the off-axis estimates provide information that would otherwise be discarded, and if the estimation errors are independent, then averaging the one on-axis and three off-axis results for each gyro should yield a better estimate. This approach improved the worst-case scale factor calibration accuracy relative to the large slew results from $6460 \mathrm{ppm}$ and $4083 \mathrm{ppm}$ for the December 11 and 16 tests, respectively, to $2117 \mathrm{ppm}$ and $571 \mathrm{ppm}$. These results were solidly within the goal of $5000 \mathrm{ppm}$ and also within the expectations from pre-launch simulations.

The team noticed and investigated unexpected features in the square wave dither gyro data. Figure 4 shows a zoomed view of the three plateaus circled in Fig. 3, which are typical of all of the plateaus in the two square wave dither tests. While the non-repeatable plateau variations are only a few microradians, note that the $5000 \mathrm{ppm}$ goal for dither calibration accuracy is equal to 0.75 microradians for the excitation level in this run of the test. These features appeared in the integrated gyro rate data but not in the star tracker data. A thorough investigation, the details of which are beyond the scope of this paper, determined that the root cause of the plateau to plateau variations was IMU and star tracker heater switching. Lockheed Martin developed changes to the heater control settings that reduced these effects, though the primary intent was improving INR performance for ABI and GLM. In parallel, a change to dither excitation was sought in order to reduce vulnerability to the artifacts seen in the 500-second square wave.

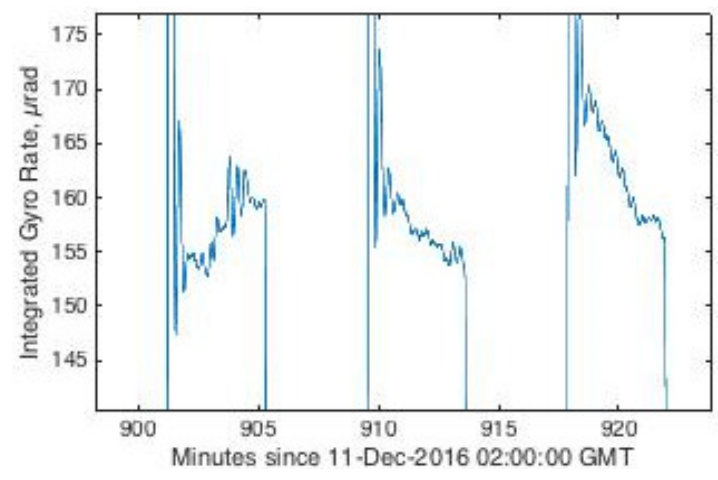

Fig. 4 Non-repeatable step response artifacts.

\section{B. Quasi-Sinusoidal Excitation}

The next improvement to the implementation of dither calibration on GOES-16 was to generate a quasi-sinusoidal excitation profile while retaining the square wave RTS scheme previously described. Figure 5 shows an overlay of the overshoot portion of twenty-five step responses from the square wave dither tests, showing a damped sinusoid response with good repeatability. The quasi-sinusoidal approach commands alternately positive and negative steps such that only the first overshoots in each step response are seen. A period of 24 seconds was empirically determined by examination of the square wave dither data as yielding a reasonable approximation of a sinusoid. 


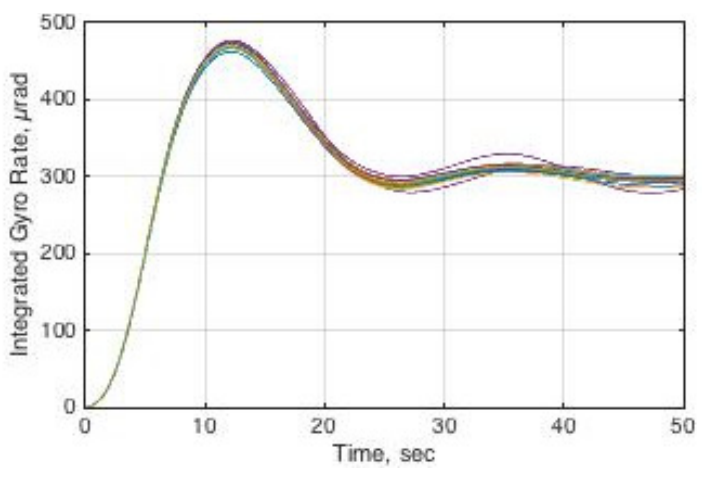

Fig. 5 Overlay of 25 step responses.

Figure 6 shows the quasi-sinusoidal response obtained from GOES-16 for a square wave command profile with a period of 24 seconds, overlaid with a sine wave of the same period and amplitude, along with the deviation of the quasi-sinusoid from the sine wave. This waveform is a reasonable approximation of the ideal sinusoidal dither excitation, with a maximum error about $8 \%$. The 24 -second quasi-sinusoidal profile avoids the plateau and overshoot artifacts and has an additional benefit over the initial square wave tests. Since dither calibration error varies approximately inversely as the square root of the number of dither periods processed, a profile with 24-second period requires about $80 \%$ less test time compared to the 500-second period for comparable accuracy.
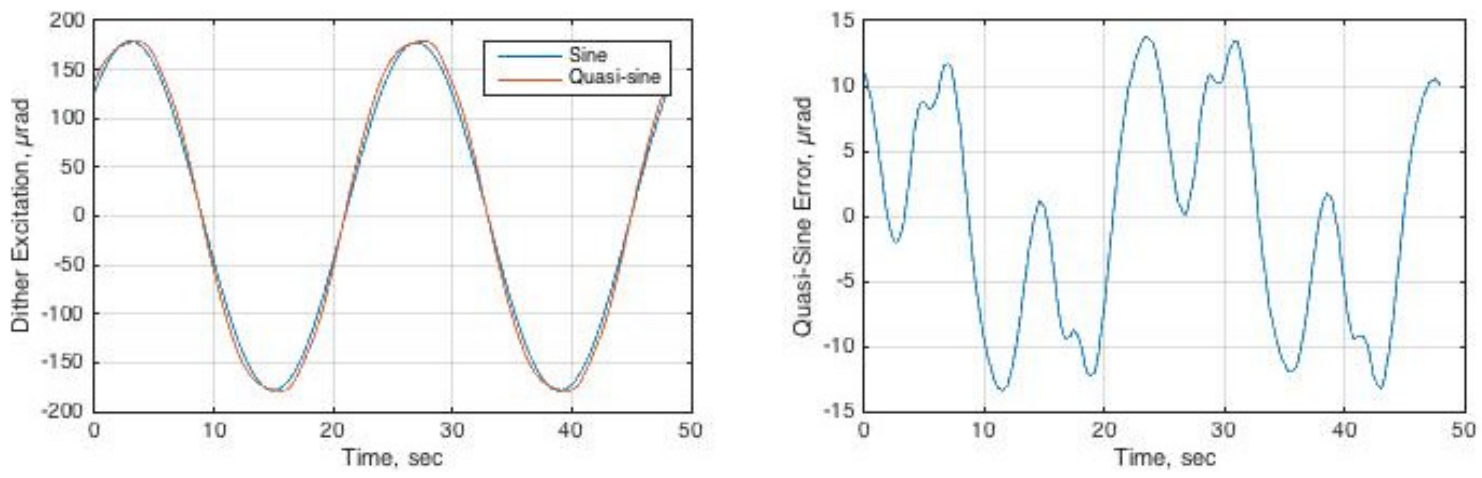

Fig. 6 GOES-16 quasi-sinusoidal response and error relative to a pure sine.

Table III summarizes the run conditions and calibration error for all dither calibration tests performed on GOES16 from launch to early 2018.

Table III Summary of GOES-16 Dither Calibration Results

\begin{tabular}{ccccccc}
\hline Test Date & Waveform & $\begin{array}{c}\text { Amplitude } \\
\boldsymbol{\mu r a d}\end{array}$ & $\begin{array}{c}\text { Period } \\
\text { sec }\end{array}$ & $\begin{array}{c}\text { Duration } \\
\text { hr }\end{array}$ & Dither Axis & $\begin{array}{c}\text { Worst-Case Error } \\
\text { ppm }\end{array}$ \\
\hline $12 / 11 / 2016$ & square & 150 & 500 & 14.5 & each gyro (4) & 2117 \\
$12 / 16-17 / 2016$ & square & 150 & 500 & 14.5 & each gyro (4) & 571 \\
$5 / 1 / 2017$ & quasi-sine & 480 & 24 & 1 & s/c yaw axis & 885 \\
$5 / 1 / 2017$ & quasi-sine & 450 & 24 & 1 & s/c yaw axis & 941 \\
$5 / 3 / 2017$ & quasi-sine & 460 & 24 & 1 & s/c yaw axis & 765 \\
$5 / 5 / 2017$ & quasi-sine & 460 & 24 & 1 & s/c yaw axis & 850 \\
$6 / 13 / 2017$ & quasi-sine & 200 & 24 & 4 & each gyro (4) & 1174 \\
$6 / 15 / 2017$ & quasi-sine & 330 & 24 & 1 & s/c yaw axis & 893 \\
$9 / 29 / 2017$ & quasi-sine & 330 & 24 & 1 & s/c yaw axis & 1075 \\
$11 / 14 / 2017$ & quasi-sine & 180 & 24 & 4 & s/c yaw axis & 577 \\
$3 / 23 / 2018$ & quasi-sine & 180 & 24 & 3.9 & s/c yaw axis & 1059 \\
\hline
\end{tabular}

Rather than exciting each gyro axis one at a time, the strategy was shifted to impart dither excitation about the spacecraft yaw axis. Referring back to Table II, the gyro arrangement in the SSIRU unit and the placement of the 
SSIRU on the spacecraft result in motion about the spacecraft yaw axis having equal projections of $1 / \sqrt{3}$ onto each of the four gyro axes. To maintain constant calibration accuracy, dither amplitude must be increased by a factor of at least $\sqrt{3}$, since dither calibration error varies approximately inversely with amplitude. For the remainder of GOES-16 PLT and Extended Validation, dither calibration used quasi-sinusoidal excitation almost always about the yaw axis.

An initial excitation amplitude of 450 microradians was selected for yaw axis dither, which is larger than the spacecraft attitude control requirements allow. However, the ABI and GLM are relatively insensitive to yaw motion, with the maximum impact occurring for lines of sight near the Earth's limb where the error is effectively attenuated by approximately $\sin 8.7^{\circ}=0.15$. Furthermore, ABI compensates for spacecraft attitude motion in two ways. First, $\mathrm{ABI}$ stabilizes its line of sight by using the spacecraft gyro rate signal as a feedforward input into its two-axis scanner control system. Secondly, ABI image navigation and registration ground algorithms also correct for spacecraft attitude motion by way of the gyro rate data. GLM's line of sight is fixed with respect to the spacecraft, but its lightning event navigation ground processing algorithms also use the spacecraft gyro data to correct for attitude motion. For these reasons, yaw dither does not degrade geolocation of ABI or GLM navigation accuracy, even though its amplitude exceeds allowable attitude control error.

There is, however one detrimental effect of higher amplitude dither on GLM. Attitude motion in conjunction with high contrast boundaries in the Earth scene causes false lightning events to be sensed by GLM. GLM ground algorithms are able to filter out false events from legitimate lightning events; however, at a certain level, the false event rate can consume the entire GLM downlink allocation, preventing some percentage of true events from being downlinked. For this reason, yaw dither calibrations using motions that are larger than specifications are performed near local midnight, when dither-induced GLM false event rates are negligible.

Although the onboard gyro scale factors have not been precisely recalibrated using large angle slews and uploaded to the spacecraft since May 2017, we assume that the gyro scale factors are essentially unchanged from that time through the most recent test reported here. The implication of this assumption is that the true gyro scale factor errors are zero and that the dither calibration results themselves are in fact dither calibration errors. This assumption will gradually break down as the true scale factors drift over mission life. Figure 7 shows the dither calibration errors for each gyro for every run of gyro scale factor dither calibration on GOES-16 using quasi-sinusoidal excitation. Of the 36 calibration errors, all but three were less than $\pm 1000 \mathrm{ppm}$, and the worst case is below $1200 \mathrm{ppm}$, significantly better than the $5000 \mathrm{ppm}$ goal.

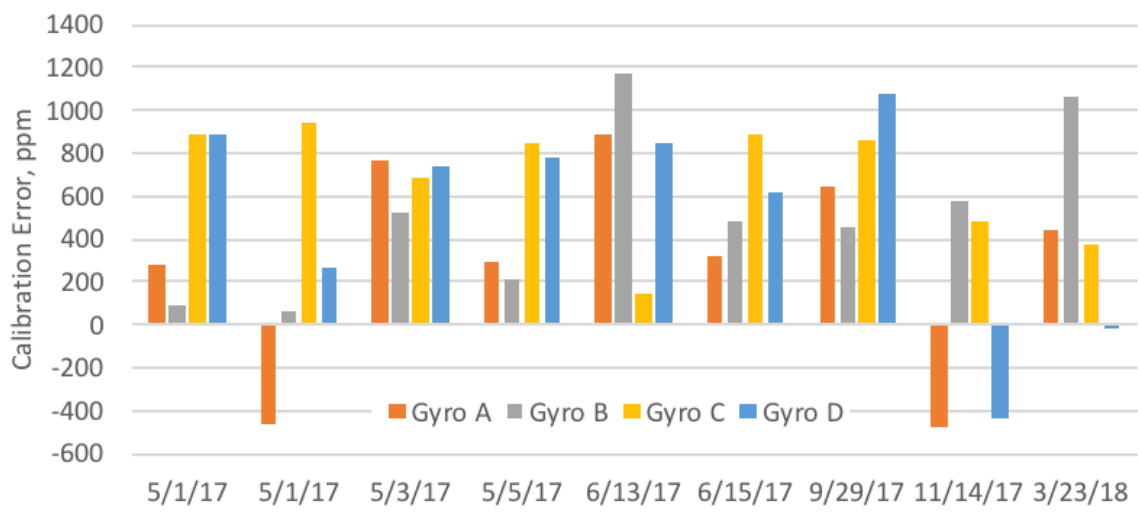

Fig. 7 GOES-16 Gyro scale factor dither calibration errors.

For the November and March test runs, the excitation level was reduced to comply with attitude control requirements, with the duration extended to theoretically yield approximately constant calibration accuracy compared to the two runs just prior. Table IV provides key spacecraft attitude and pointing requirements for Earth and Sun pointing instruments with performance achieved during the November 14, 2017 dither test also indicated. Although the requirement for sun pointing stability is violated during dither excitation, the actual need for image quality is pointing stability over the longest exposure time of the SUVI, which is currently one second. Estimated performance with respect to one-second sun pointing stability is 2.15 arc-seconds, based on the peak rate of change observed in the sun pointing radial error in $0.25 \mathrm{~Hz}$ telemetry. One-second sun pointing stability is shown in italics in Table IV since it is not the requirement, though it more accurately captures the performance currently needed for SUVI science data quality than the 60 -second stability requirement. 
Table IV Requirements Compliance During Dither

\begin{tabular}{|c|c|c|}
\hline & Specification & Performance \\
\hline \multicolumn{3}{|l|}{ Earth-Pointing Instruments } \\
\hline Attitude control & $< \pm 360 \mu \mathrm{rad}$ & $\pm 180 \mu \mathrm{rad}$ \\
\hline Attitude rate error & $< \pm 100 \mu \mathrm{rad} / \mathrm{sec}$ & $\pm 60 \mu \mathrm{rad} / \mathrm{sec}$ \\
\hline Attitude stability, $60 \mathrm{sec}$ & $<500$ urad, p-p & $360 \mu \mathrm{rad}$ \\
\hline \multicolumn{3}{|l|}{ Sun-Pointing Instruments } \\
\hline Pointing control & $< \pm 90$ arc-sec & \pm 10 arc-sec \\
\hline Pointing stability, $60 \mathrm{sec}$ & $<6$ arc-sec, radial & 12 arc-sec \\
\hline Pointing stability, $1 \mathrm{sec}$ & $<6$ arc-sec, radial & 2.2 arc $-\mathrm{sec}$ \\
\hline \multicolumn{3}{|l|}{ GN\&C } \\
\hline Wheel torque, per wheel & $< \pm 0.35 \mathrm{Nms}$ & $\pm 0.12 \mathrm{Nms}$ \\
\hline
\end{tabular}

\section{GOES-16 Operations and Beyond}

Lockheed Martin estimated the drift rate of gyro scale factors based on large slew gyro calibrations performed near the beginning and end of the six-month PLT phase and recommended that scale factor recalibrations be performed every five years. The baseline approach for scale factor recalibration uses 3.5 degree spacecraft slews about each gyro axis. While smaller than the 19 degree slews used for high-accuracy calibration during PLT, these slews are disruptive nonetheless, taking GOES-16 instruments offline for about an hour and resulting in ABI INR degradation for about 30 hours. Since the dither method for gyro scale factor calibration was proven and performed better than threshold requirements and goals, it has been baselined for GOES-16 operations for scale factor trending but not recalibration. Although dither scale factor calibration has an accuracy on the order of $1000 \mathrm{ppm}$, small slew calibrations were shown during PLT to have an accuracy approximately an order of magnitude better.

Dither calibrations will be performed twice per year, near midnight near the equinoxes when the spacecraft yaw axis is nearly aligned with the sun vector, thus reducing coupling of the yaw dither motion into solar instrument pointing. The results from the semiannual dither calibrations will be trended to detect either: a) that gyro scale factors are more stable than expected, and small slew recalibrations may be postponed, thus deferring the associated operational downtime, or b) that gyro scale factors are drifting faster than expected, and small slew recalibration is warranted sooner than five years. In other words, dither calibration is being used as a non-disruptive means of monitoring gyro scale factors and possibly driving rescheduling of the baselined quinquennial small slew recalibrations. Baseline parameters for the semiannual dither calibrations are the same as the November 2017 and March 2018 calibrations shown in the last rows of Table III.

At the request of the GOES-R Flight Project, Lockheed Martin added functionality to the GN\&C flight software to generate dither excitation onboard for GOES-S, -T, and -U. The dither generator is controlled by two new spacecraft commands, one to set parameters and the other to activate and deactivate the dither profile. Commanded parameters comprise: amplitude, period, test duration in units of number of periods, eigenvector, and torque gain. Eigenvector specifies the dither axis in the attitude control system reference frame. Torque gain is nominally unity and is in series with a dither torque feedforward term that aids attitude control system tracking of the dither profile. The dither generator generates at a $20 \mathrm{~Hz}$ update rate a transformation matrix that adjusts the two aforementioned body reference vectors that the attitude control system aligns with nadir and negative orbit normal during normal operations.

With onboard dither generation, GOES-17 operations are simplified relative to GOES-16 dither testing, with the elimination of RTS uploads and a great reduction in the number of commands being logged and echoed to the ground, currently 300 per hour with the baseline profile. In addition, the dither generator produces a sine wave profile, which is more harmonically pure than the quasi-sinusoidal profile, as shown in Fig. 8. The sine profile on GOES-17 is also expected to be more benign in terms of attitude accelerations than the square wave commanding used on GOES-16.

Table V Summary of GOES-17 Dither Calibration Results

\begin{tabular}{ccccccc}
\hline Test Date & Waveform & $\begin{array}{c}\text { Amplitude } \\
\boldsymbol{\mu r a d}\end{array}$ & $\begin{array}{c}\text { Period } \\
\text { sec }\end{array}$ & $\begin{array}{c}\text { Duration } \\
\text { hr }\end{array}$ & Dither Axis & $\begin{array}{c}\text { Worst-Case Error } \\
\text { ppm }\end{array}$ \\
\hline $3 / 27 / 2018$ & quasi-sine & 180 & 24 & 4 & s/c yaw axis & 1440 \\
\hline
\end{tabular}



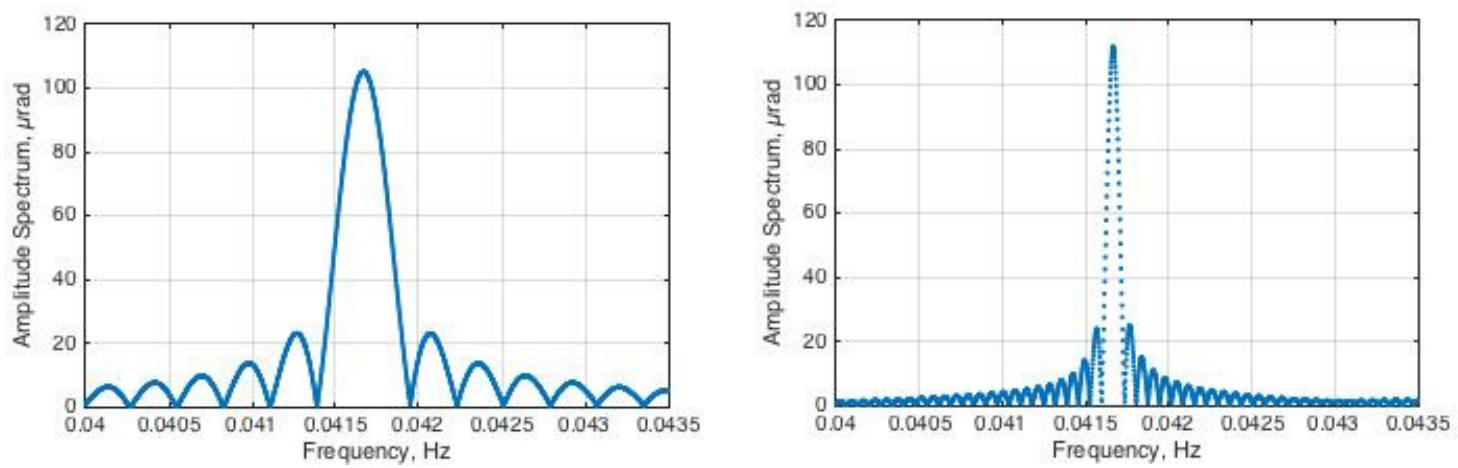

Fig. 8 Comparison of amplitude spectra of quasi-sinusoidal vs sinusoidal excitation.

Dither gyro scale factor calibration using onboard dither generation is being tested on GOES-17, which was launched as GOES-S on March 1, 2018 and renamed upon reaching geosynchronous orbit on March 12. As on GOES16 , these tests use the large slew calibration results as the truth reference. Initial testing on GOES-17 use the same baseline dither parameters as the November 2017 and March 2018 GOES-16 calibrations; however, the flight software was updated to compute a true sinusoidal dither profile as shown in Fig. 9. Table V shows the results of the first dither calibration test on GOES-17 using onboard dither generation.

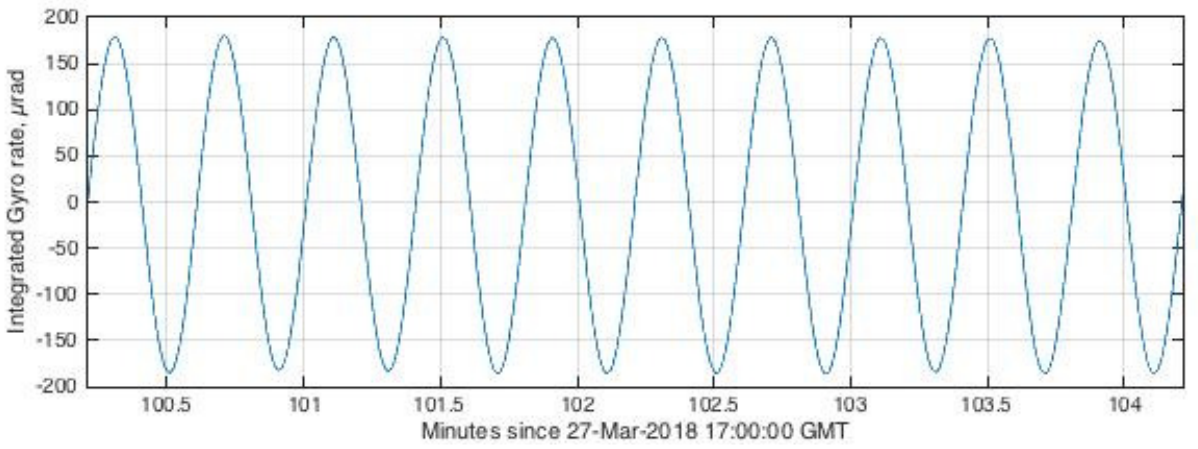

Fig. 9 Measured GOES-17 sine wave dither excitation.

The GOES-R Project GN\&C team will investigate why this initial result appears to be worse than the typical accuracy from GOES-16 dither calibrations. Subsequent testing during GOES-17 PLT will seek to maintain or improve calibration accuracy while reducing dither amplitude, with a goal of eliminating constraints on time of day due to GLM false events concerns and time of year constraints due to coupling into solar instrument pointing. Findings from GOES-17 PLT may be transferred to GOES-16 operations to move closer to the ideal goal of completely unobtrusive calibration suitable for trending gyro scale factor over mission life.

\section{Conclusion}

Dither gyro scale factor calibration is a novel approach to calibration, offering increased operational availability and simplified mission operations over more traditional techniques. Its small dither motion is within pointing requirements avoiding the need to take the instruments offline, reducing the burden on the mission operations team. As demonstrated with the GOES-16 spacecraft on-orbit, the dither gyro scale factor calibration technique achieves this without sacrificing accuracy, estimating gyro scale factor errors to within $1500 \mathrm{ppm} 3$-sigma. Results were so promising that the FSW on subsequent spacecraft in the series was modified to include an algorithm to generate a true sinusoidal dither profile. The GOES-17 spacecraft and beyond will also use this dither profile routinely to trend scale factor error throughout mission life. Dither gyro scale factor calibration is Patent Pending by Chesapeake Aerospace, Grasonville, MD, USA. 


\section{Acknowledgments}

The authors gratefully acknowledge the support of the GOES-R Flight Project, GOES-R Mission Operations Support Team (MOST) in particular Frank Liu and Jess Endicter; Lockheed Martin Space Systems Denver in particular Brian Clapp, Tim Bevacqua and Jim Chapel; and the NOAA Office of Satellite and Product Operations (OSPO)/GOES Engineering in particular Paul Quiroga, Brian Middleton, and Zachary Harlow in testing and demonstrating the dither gyro scale factor calibration method. This work was sponsored by the NASA Goddard Space Flight Center under contracts NNG15CR65C and NNG09HR00C.

\section{References}

[1] Reth, A., Chu, D., Lorenz, D., Freesland, D., Stancliffe, D., Clapp, B., Chapel, J., Cwynar, D., Krimchansky, A., "GOES-R Gyro Scale Factor Calibration," 47 $7^{\text {th }}$ Annual AAS Guidance and Control Conference, AAS 11-095, Breckenridge, CO, USA, February 2011.

[2] Chapel, J., Stancliffe, D., Bevacqua, T., Winkler, S., Clapp, B., Rood, T., Gaylor, D., Freesland, D., Krimchansky, A, "Guidance, Navigation, and Control Performance for the GOES-R Spacecraft," CEAS Space Journal, DOI 10.1007/s12567015-0077-1, March 2015.

[3] Chapel, J., Stancliffe, D., Bevacqua, T., Winkler, S., Clapp, B., Rood, T., Freesland, D., Reth, A., Early, D., Walsh, T., Krimchansky, A, "In-Flight Guidance, Navigation, and Control Performance Results for the GOES-16 Spacecraft," $10^{\text {th }}$ International ESA Conference on Guidance, Navigation \& Control Systems, Salzburg, Austria, June 2017.

[4] Freesland, D., Chu D., Reth, A, Krimchansky, A., Donnelly, M., Comeyne, G., "Advancing the Next Generation GOES-R Operational Availability," $41^{\text {st }}$ Annual AAS Guidance and Control Conference, AAS 05-006, Breckenridge, CO, USA, February 2005.

[5] Carter, D., Clapp, B., Early, D., Freesland, D., Chapel, J., Bailey, R., Krimchansky, A, “GOES-16 On-Orbit Dual Isolation Performance Characterization Results," $10^{\text {th }}$ International ESA Conference on Guidance, Navigation \& Control Systems, Salzburg, Austria, June 2017.

[6] Clapp, B., Weigl, H., Goodzeit, N., Carter, D., Rood, T., "GOES-R Active Vibration Damping Controller Design, Implementation and On-Orbit Performance," 10 $10^{\text {th }}$ International ESA Conference on Guidance, Navigation \& Control Systems, Salzburg, Austria, June 2017.

[7] Winkler, S., Ramsey, G., Frey, C., Chapel, J., Chu, D., Freesland, D., Krimchansky, A., Concha, M., "GPS Receiver On-Orbit Performance for the GOES-R Spacecraft," $10^{\text {th }}$ International ESA Conference on Guidance, Navigation \& Control Systems, Salzburg, Austria, June 2017. 